\title{
Validación de encuesta de calidad de vida "SNOT-22" en población chilena
}

\section{Validation of the SNOT-22 quality of life survey in Chilean population}

\author{
Constanza Valdés P. ${ }^{1,2}$, Tamara Muñoz M. ${ }^{1}$, Tamara Barría E. ${ }^{1,3}$, Mariana Bogado B. ${ }^{4}$, \\ Paula Ruz M. ., , Felipe Cardemil M..${ }^{2,6}$, Samanta Ruz G. ${ }^{1,7}$, Marcela Veloz T. ${ }^{1,5}$
}

\section{Resumen}

Introducción: La rinosinusitis crónica (RSC) tiene un alto impacto en la calidad de vida del paciente. La recomendación de las guías EPOS es utilizar la encuesta Sino-Nasal-Outcome-Test de 22 indicadores (SNOT-22), la cual no está validada en población chilena. Objetivo: Traducción y validación de SNOT-22 en población chilena consultante por RSC en el Servicio de Otorrinolaringología del Hospital del Salvador. Material y Método: Traducción de SNOT-22, reclutamiento de pacientes con y sin RSC. Aplicación de versión traducida en dos ocasiones con dos semanas de diferencia. Evaluación de validez de constructo y discriminante. Resultados: Se evaluaron 34 pacientes sin RSC y 16 con RSC. El puntaje total promedio de SNOT-22 fue 19,62 y 43,1 puntos, con diferencias significativas entre ambos grupos. Respecto a la validación, se observó un coeficiente alfa Cronbach $>0,95$, un coeficiente de correlación intraclase de 0,986, un coeficiente de correlación de Pearson de 0,84 y un área bajo la curva ROC de 0,861. Conclusión: La traducción y adaptación transcultural de esta versión de SNOT-22 es válida y confiable. Esta versión de SNOT-22 es un buen instrumento para evaluar el impacto de RSC y su intervención en la calidad de vida de pacientes adultos chilenos. Palabras clave: encuesta de calidad de vida, SNOT-22, rinosinusitis crónica.

\begin{abstract}
Introduction: Chronic rhinosinusitis has a high impact on the patient's quality of life. The recommendation of the EPOS guidelines is to use the Sino-Nasal-Outcome-Test survey of 22 indicators (SNOT-22), which is not validated in the Chilean population. Objective: translation and validation of SNOT-22 in Chilean population consulting for CRS in the ENT department of the Hospital del Salvador. Material and Method: translation of SNOT-22. Recruitment of patients with and without CRS. Application of translated version two times two weeks apart. Evaluation of discriminant validity and construct validity. Results: 34 patients without CRS and 16 with CRS were evaluated. The average total SNOT-22 score was 19.62 and 43.1 points, respectively, with significant differences between both groups. Regarding validation analysis, Cronbach's alpha coefficient observed was $>0.95$, intraclass correlation coefficient was 0.986, Pearson's correlation coefficient was 0.84 and the area under the ROC curve was 0.861. Conclusion: The translation and transcultural adaptation of this version of SNOT-22 is valid and reliable. This version of SNOT-22 is a good instrument to assess the impact of CSR, and its intervention, on the quality of life of Chilean adult patients.
\end{abstract}

Keywords: quality of life survey, SNOT-22, chronic rhinosinusitis.

\author{
'Servicio de \\ Otorrinolaringología Hospital \\ del Salvador, Universidad de \\ Chile. Santiago, Chile. \\ ${ }^{2}$ Servicio de \\ Otorrinolaringología, Clínica \\ Las Condes. Santiago, Chile. \\ ${ }^{3}$ Servicio de \\ Otorrinolaringología Clínica \\ Redsalud Providencia. \\ Santiago, Chile. \\ ${ }^{4}$ Servicio Otorrinolaringología \\ Hospital San Juan de Dios, La \\ Serena. Chile. \\ ${ }^{5}$ Servicio de \\ Otorrinolaringología Clínica \\ Santa María. Santiago, Chile. \\ ${ }^{6}$ Departamento de \\ Otorrinolaringología, \\ Universidad de Chile. \\ Santiago, Chile. \\ ${ }^{7}$ Servicio de \\ Otorrinolaringología Clínica \\ Indisa. Santiago, Chile. \\ Los autores declaran no tener \\ conflictos de interés. \\ Recibido el 4 de marzo de \\ 2020. Aceptado el 29 de julio \\ de 2020. \\ Correspondencia: \\ Constanza Valdés $P$. \\ Avenida Salvador \#364, \\ Providencia. \\ Santiago, Chile. \\ Email: cvaldesp@clc.cl
}

\section{Introducción}

La rinosinusitis crónica (RSC) se define como la inflamación de la nariz y cavidades perinasales por más de 12 semanas, caracterizada por dos o más síntomas cardinales (obstrucción/congestión o descarga nasal). Además, deben haber signos endoscópicos (pólipos nasales, descarga mucopurulenta desde el meato medio y/o edema/obstrucción mucosa del meato medio) o cambios en la tomografía computada (cambios mucosos 
en el complejo osteomeatal y/o en los senos paranasales $)^{1}$. Se estima que alrededor de $5 \%$ a $15 \%$ de la población general en Europa y Estados Unidos está afectada por RSC ${ }^{2-5}$. Esta patología tiene un alto impacto en la calidad de vida $(\mathrm{CdV})$ de los pacientes. No sólo por la sintomatología nasosinusal, sino porque también afecta la calidad del sueño, autoestima y sensación de falta de energía para las actividades cotidianas ${ }^{6}$. Esto se ve reflejado en los puntajes de las encuestas de $\mathrm{CdV}$ donde los pacientes con RSC, no sólo tienen puntajes menores respecto de la población normal, sino que puntajes inferiores a pacientes con enfermedades invalidantes como la insuficiencia cardíaca, angina o dolor de espalda ${ }^{7}$.
Por consiguiente, es importante contar con instrumentos validados que permitan no sólo objetivar los síntomas y su respectivo impacto en la calidad de vida del paciente, sino además, monitorear la efectividad del tratamiento y realizar un seguimiento objetivo del paciente y su enfermedad.

Existen diversas herramientas para la evaluación de la $\mathrm{CdV}$ en pacientes adultos con RSC. La recomendación de las guías EPOS $2012^{1}$ es la utilización de la encuesta Sino-Nasal Outcome Test de 22 indicadores (SNOT-22). El SNOT-22 (Figura 1) es un cuestionario de autoaplicación, derivado de la modificación de la encuesta SNOT- $20^{8}$, en que la puntuación se ha simplificado y se agregaron dos medidas:
Figura 1. Versión original de Sino-Nasal Outcome Test de 22 indicadores.

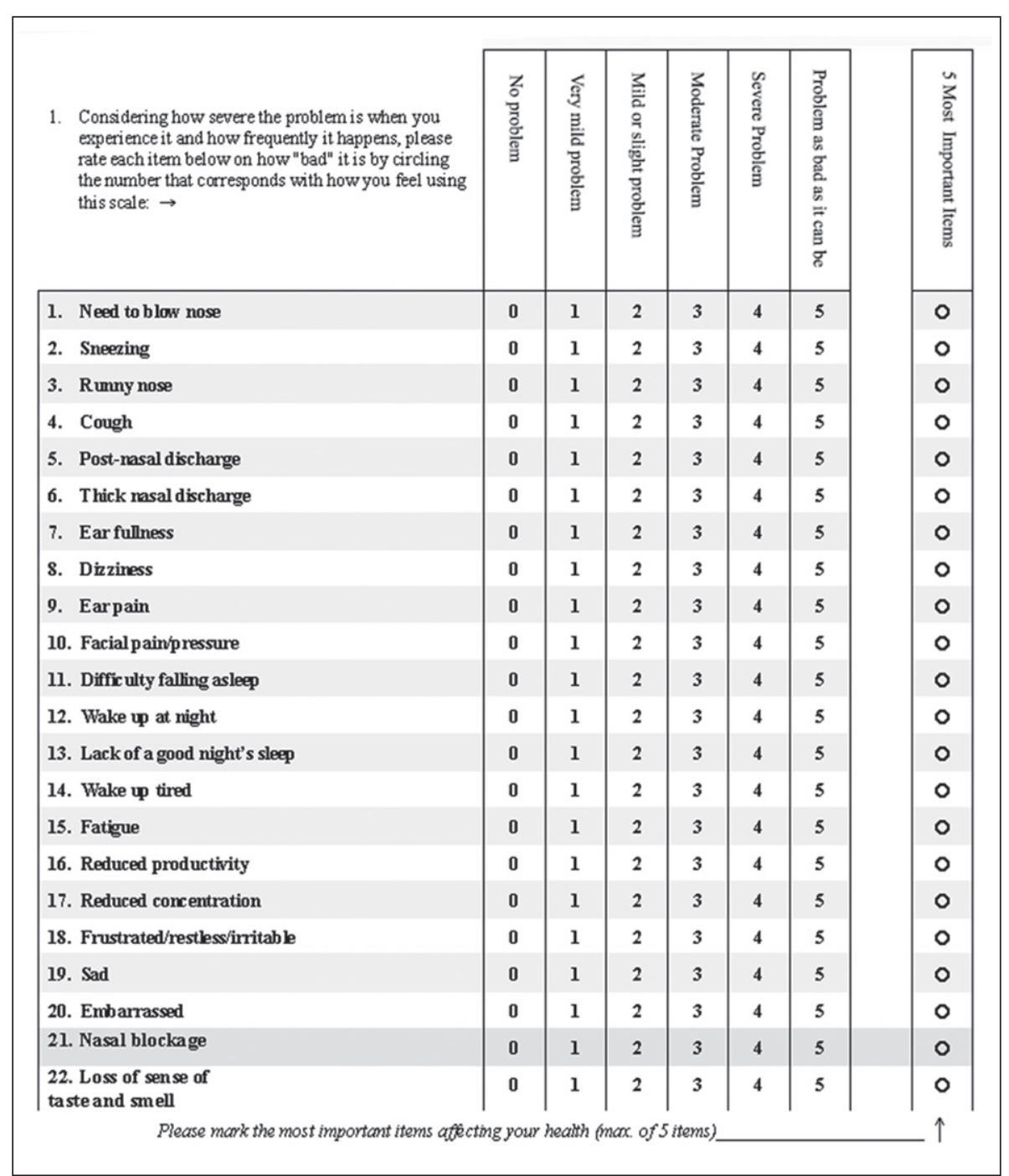


obstrucción nasal y alteraciones del olfato y gusto $^{8,9}$. La encuesta tiene la ventaja de que, además de evaluar la sintomatologóa nasosinusal, contempla al individuo en forma integral con las repercusiones físicas, funcionales y emocionales que le genera la enfermedad. Este cuestionario se encuentra validado en diversos idiomas incluido el español ${ }^{8,10,19-25}$, pero no existe la versión validada en población chilena.

\section{Objetivo}

El objetivo del presente trabajo es la traducción y validación de la encuesta SNOT-22 en población chilena.

\section{Metodología}

Estudio de corte transversal de validación de instrumento. Este estudio cuenta con la aprobación del Comité de Ética-Científico Servicio de Salud Metropolitano Oriente. El proyecto no está financiado por fuentes externas, ni existen conflictos de interés que declarar por parte de los autores del mismo.

\section{Traducción y adaptación transcultural de encuesta}

Se realiza la traducción directa o traducción conceptual del instrumento original a través de la traducción al español por dos profesionales del área otorrinolaringológica bilingües independientes, siguiendo las guías internacionalmente aceptadas ${ }^{26}$. Luego, se obtiene un consenso entre ambas versiones. A continuación se realiza la traducción inversa al inglés por un tercer profesional bilingüe, ciego a la versión original y se comparó con esta última. Posteriormente, se realiza una prueba piloto con la versión traducida en sujetos con diagnóstico de RSC, con la instrucción de comentar cualquier aspecto que haya resultado difícil de entender, para evaluar la aplicabilidad $\mathrm{y}$ viabilidad del cuestionario.

\section{Reclutamiento y evaluación de sujetos}

Se reclutan pacientes chilenos atendidos consecutivamente en el Servicio de Otorrinolaringología del Hospital del Salvador. Los pacientes se dividen en dos grupos: grupo 1: sin RSC y grupo 2: con RSC. Se consideran criterios de inclusión para el grupo 1: 1) ser adulto chileno (18 años o más); 2) que no cumple criterios EPOS 2012 para RSC; 3) con consentimiento informado firmado. Mientras que los criterios de inclusión para el grupo 2 son: 1) ser adultos chilenos (18 años o más); 2) con diagnóstico de RSC, según criterios EPOS 2012;3) con consentimiento informado firmado.

Los pacientes completan la versión traducida consensuada de la encuesta SNOT-22. Luego de contestar la encuesta se les pregunta si tuvieron dudas sobre el cuestionario. Se realiza una segunda evaluación a las dos semanas, vía telefónica o presencial, según preferencia del paciente. No se proporciona tratamiento al paciente durante este período de transición entre ambas evaluaciones. Se recolecta la información clínica y demográfica del paciente: edad, género, nivel educacional, diagnóstico, características a la endoscopía nasal y puntaje Lund-Mackay de tomografía computada. Ningún paciente recibió pago o compensación por la participación en el protocolo.

\section{Análisis estadístico}

El análisis estadístico se realizó con el software estadístico STATA 13. Para los datos descriptivos continuos se calculan los valores medios y su desviación estándar, y porcentajes para las variables categóricas. Comparación de grupos mediante prueba de $\chi^{2}$, t-student y prueba de Mann-Whitney.

\section{Validación del cuestionario traducido}

Se evalúa la validez aparente (lo que el cuestionario parece medir) y validez de contenido o grado en que el test presenta una muestra adecuada de los contenidos a los que se refiere ${ }^{27,28}$, considerando las opiniones de los autores y expertos, el estudio piloto y los comentarios de los participantes.

Para garantizar la preservación de las propiedades psicométricas del instrumento original, se evaluó su fiabilidad en dos dimensiones o validez de constructo, definido como grado de correspondencia entre las variables observadas y el constructo teórico que se quiere medir ${ }^{27}$. Primero, se evaluó la consistencia interna o estabilidad del instrumento, el cual aplicado repetidas veces debe presentar resultados consistentes, o bien, las 
distintas partes que componen el instrumento estén midiendo lo mismo ${ }^{27-29}$, esto se determinó mediante el cálculo del coeficiente alfa de Cronbach, utilizando como valor suficiente para garantizarla determinado en un valor alfa superior a 0,7. Luego, se evaluó la fiabilidad intraobservador o test-retest, donde la aplicación del instrumento en los mismos fenómenos y condiciones debería arrojar resultados congruentes $^{27}$, repitiendo la aplicación del cuestionario con dos semanas de diferencia entre la primera y segunda evaluación, calculando el coeficiente de correlación intraclase mediante análisis de varianza (ANOVA) de mediciones repetidas, donde consideramos una buena correlación con valores superiores a 0,7 . A continuación, se evaluó la validez concurrente a través de comparación de puntajes totales y de cada pregunta por separado, utilizando el coeficiente $\mathrm{R}$ de Pearson, y se procedió a evaluar la validez convergente para evaluar correlación entre categorías similares y diferentes ${ }^{28,29}$, a través de análisis factorial exploratorio y medición de la sensibilidad al cambio o capacidad que tiene un instrumento de detectar cambios en los atributos o sujetos ${ }^{30,31}$, mediante effect size (siendo significativo mayor $>0,8$ ) entre la primera y segunda aplicación del instrumento traducido dentro de un mismo grupo. Finalmente, se realiza un estudio de validez discriminante o capacidad de distinguir entre individuos que se espera que sean diferentes ${ }^{30,31}$, mediante curvas ROC, utilizando como discriminante pertenecer al grupo con o sin patología inflamatoria rinosinusal.

\section{Resultados}

Después de la traducción, traducción inversa, revisión y adaptación cultural, se obtuvo una versión final del SNOT 22 en español, que se detalla en la Figura 2. En la prueba piloto, no hubo reclamos respecto a su comprensión, ni solicitud de aclaración de ninguna pregunta evaluada. Inicialmente se reclutaron 64 sujetos, 42 sin RSC y 22 pacientes con RSC. Sin embargo, para la re-aplicación del test se logró evaluar a 50 pacientes, 34 sin RSC y 16 con RSC. De estos, el $60 \%$ son mujeres. La mediana de edad total fue de 24 años, con un mínimo de
23 y un máximo de 67 años. Entre los grupos 1 y 2 hubo diferencias significativas en términos de edad, pero no respecto al género. En cuanto a la validez aparente, el cuestionario SNOT-22 ha sido validado y recomendado ampliamente por numerosos comités de expertos. Además, los pacientes reclutados consideraron este cuestionario pertinente, indicando la validación de contenido.

El grupo sin RSC tuvo un promedio de $19,62$ puntos ( $D S=14,18)$ en la encuesta traducida, mientras que en el grupo con RSC fue de 43,1 puntos (DS $=16,5$ ) (Figura 3 ), existiendo diferencias significativas entre ambos grupos para la primera aplicación del SNOT-22 traducido (Wilcoxon rank-sum; valor $p 0,0004$ ) y la segunda aplicación (Wilcoxon rank-sum; valor $p$ 0,0092), pero al evaluar cada grupo por separado, comparando con sí mismo, no hubo diferencias significativas entre los puntajes de la primera y la segunda aplicación (T-Student; valor $p$ 0,1531).

Respecto a la validez de constructo, la consistencia interna mostró un coeficiente alfa de Cronbach inter-ítem de 0,95 para el test, y de 0,96 en la etapa re-test, con un coeficiente de correlación de Pearson de 0,84 . En cuanto a la estabilidad temporal o concordancia de las dos aplicaciones separadas por 2 semanas, el coeficiente de correlación intraclase fue 0,986. Además, a la evaluación del coeficiente de correlación de Pearson para cada pregunta por separado, resulta una correlación mayor a 0,6 para todas las preguntas. En todos los casos, observamos una correlación positiva (Tabla 1).

Se evalúa la posibilidad de realizar un análisis factorial exploratorio para evaluar validez convergente, sin embargo, no se encuentra normalidad multivariada y no es posible proseguir con el análisis por componentes principales.

En cuanto a la sensibilidad al cambio, que evalúa los cambios en los puntajes entre la primera y la segunda medición, se aprecia una gran Effect size de 0,928. En cuanto a la validez discriminante, se evalúa el instrumento como prueba diagnóstica obteniendo un área bajo la curva ROC de 0,861 (Figura 4), con una sensibilidad de $45,45 \%$, especificidad de $91,18 \%$, valor predictivo positivo de $62,5 \% \mathrm{y}$ valor predictivo negativo de $83,78 \%$. 


\section{CUESTIONARIO DE SINTOMAS NASOSINUSALES - SNOT-22 (SINO-NASAL OUTCOME TEST)}

Fecha:

A continuación, usted encontrará una serie de síntomas y alteraciones socio/emocionales asociadas a su rinosinusitis. Nos gustaría saber más acerca de estos problemas y le solicitamos contestar las siguientes preguntas, lo mejor posible. No hay respuestas correctas o incorrectas y sólo usted nos puede entregar esta información.

Por favor, califique sus molestias según cómo han sido estas últimas dos semanas. Gracias por su participación. No dude en pedir ayuda si la necesita.

1. Considere cuán severas son sus molestias cuándo éstas están presentes y con cuánta frecuencia se presentan, por favor, califique cada ítem a continuación en cuán "malo" o "molesto" es, rodeando con un círculo el numero que se corresponda con sus molestias, usando la siguiente escala: $\rightarrow$

\begin{tabular}{|c|c|c|c|c|c|c|}
\hline 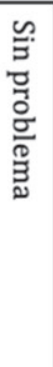 & 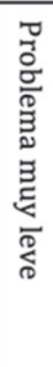 & 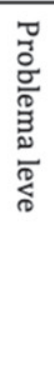 & $\begin{array}{l}0 \\
0 \\
0 \\
0 \\
0 \\
0 \\
0 \\
0 \\
0 \\
0 \\
0 \\
0 \\
0\end{array}$ & $\begin{array}{l}0 \\
0 \\
0 \\
0 \\
0 \\
0 \\
0 \\
0 \\
0 \\
0 \\
0 \\
0 \\
0\end{array}$ & 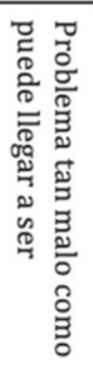 & 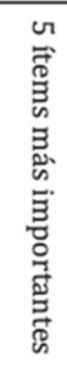 \\
\hline 0 & 1 & 2 & 3 & 4 & 5 & 0 \\
\hline 0 & 1 & 2 & 3 & 4 & 5 & 0 \\
\hline 0 & 1 & 2 & 3 & 4 & 5 & 0 \\
\hline 0 & 1 & 2 & 3 & 4 & 5 & 0 \\
\hline 0 & 1 & 2 & 3 & 4 & 5 & 0 \\
\hline 0 & 1 & 2 & 3 & 4 & 5 & 0 \\
\hline 0 & 1 & 2 & 3 & 4 & 5 & 0 \\
\hline 0 & 1 & 2 & 3 & 4 & 5 & 0 \\
\hline 0 & 1 & 2 & 3 & 4 & 5 & 0 \\
\hline 0 & 1 & 2 & 3 & 4 & 5 & 0 \\
\hline 0 & 1 & 2 & 3 & 4 & 5 & 0 \\
\hline 0 & 1 & 2 & 3 & 4 & 5 & 0 \\
\hline 0 & 1 & 2 & 3 & 4 & 5 & 0 \\
\hline 0 & 1 & 2 & 3 & 4 & 5 & 0 \\
\hline 0 & 1 & 2 & 3 & 4 & 5 & 0 \\
\hline 0 & 1 & 2 & 3 & 4 & 5 & 0 \\
\hline 0 & 1 & 2 & 3 & 4 & 5 & 0 \\
\hline 0 & 1 & 2 & 3 & 4 & 5 & 0 \\
\hline 0 & 1 & 2 & 3 & 4 & 5 & 0 \\
\hline 0 & 1 & 2 & 3 & 4 & 5 & 0 \\
\hline 0 & 1 & 2 & 3 & 4 & 5 & 0 \\
\hline 0 & 1 & 2 & 3 & 4 & 5 & 0 \\
\hline
\end{tabular}

Por favor marque los ítems que más afectan su salud (máximo 5) ^

Figura 2. Adaptación transcultural chilena de cuestionario de síntomas nasosinusales de 22 indicadores (SNOT-22). 
Figura 3. Validez concurrente. Comparación entre la primera (en azul) y segunda (en rojo) evaluación, para los grupos 1 y 2. Las cajas representan los percentiles 25 y 75 de la muestra. Las líneas dentro de la caja la mediana. Las barras los rangos máximo y mínimo.

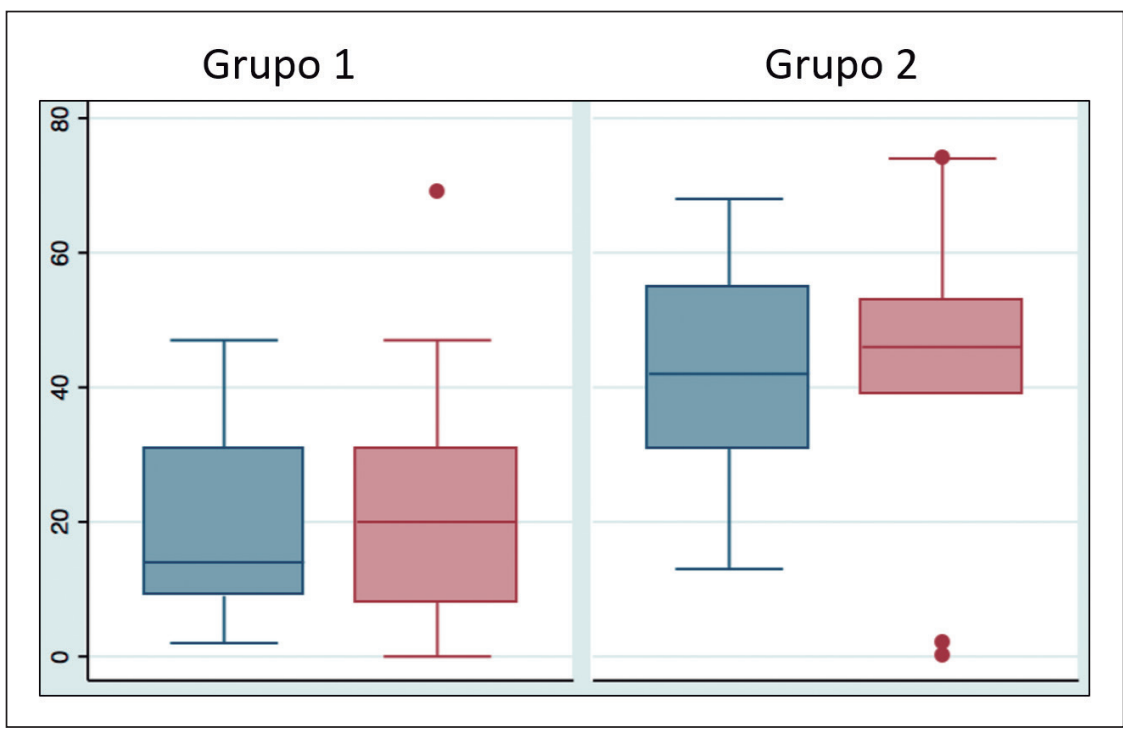

\section{Tabla 1. Correlación intraclase de cada pregunta del cuestionario adaptado}

\begin{tabular}{|c|c|}
\hline Pregunta & $\mathbf{R}$ de Pearson \\
\hline 1. Necesidad de sonarse la nariz & 0,8286 \\
\hline 2. Estornudos & 0,8078 \\
\hline 3. Secreción nasal continua & 0,8185 \\
\hline 4. Tos & 0,7739 \\
\hline 5. Sensación de moco que cae por la parte posterior de la garganta & 0,6920 \\
\hline 6. Secreción nasal espesa & 0,7892 \\
\hline 7. Sensación de oído taponado & 0,7342 \\
\hline 8. Mareo & 0,7580 \\
\hline 9. Dolor de oído & 0,6398 \\
\hline 10. Dolor / presión facial & 0,7485 \\
\hline 11. Dificultad para quedarse dormido & 0,6170 \\
\hline 12. Se despierta durante la noche & 0,6991 \\
\hline 13. Dormir mal por la noche & 0,7224 \\
\hline 14. Se despierta cansado & 0,8488 \\
\hline 15. Fatiga o cansancio & 0,8276 \\
\hline 16. Disminución en el rendimiento / productividad & 0,8697 \\
\hline 17. Disminución en la concentración & 0,8193 \\
\hline 18. Frustrado / inquieto / irritable & 0,7704 \\
\hline 19. Triste & 0,6776 \\
\hline 20. Avergonzado & 0,7504 \\
\hline 21. Alteraciones del gusto / olfato & 0,8797 \\
\hline 22. Congestión / obstrucción nasal & 0,8800 \\
\hline
\end{tabular}




\section{Discusión}

La prevalencia de RSC es alta, observándose con frecuencia en la consulta otorrinolaringológica. Al no existir tratamiento curativo, el manejo tiene como objetivo la disminución de los síntomas y control de la inflamación. Por esto, es fundamental tener un instrumento que permita unificar y objetivar la sintomatología del paciente en la consulta inicial, evaluar la efectividad del tratamiento y hacer el seguimiento en las consultas posteriores. El cuestionario SNOT-22 ha sido validado en numerosos idiomas y si bien existe una versión en español, esta no necesariamente es aplicable en una población hispanohablante latinoamericana como la chilena, dado las variantes semánticas del idioma en cada región. Si bien se mantiene la misma base fundamental que el idioma europeo, sobre todo en el nivel morfosintáctico, tenemos también un vocabulario básico fundamental común. Este, se ha visto influenciado por los cambios económicos e industriales del país, relacionados con las condiciones sociales y la influencia de la administración pública y medios de comunicación ${ }^{32,33}$.

A través de este estudio se realiza la adaptación transcultural de la encuesta de CdV recomendada por las guías europeas para el seguimiento de los pacientes con RSC. La pérdida de sujetos del estudio se explica por la inasistencia al segundo control clínico. Se ha demostrado la confiabilidad y consistencia del cuestionario traducido, cuya evaluación test-retest muestra estabilidad temporal de la prueba en un mismo paciente.

Esta versión de SNOT-22 en español, tuvo una gran magnitud de efecto respecto a la sensibilidad al cambio, por lo que es un buen instrumento para evaluar el impacto en la calidad de vida de los pacientes adultos con RSC y el impacto en ésta de alguna intervención orientada a su tratamiento. Si bien el objetivo de este instrumento es seguimiento de pacientes con RSC, no diagnóstico, nuestros datos han mostrado que es altamente probable que detecte y discrimine a los pacientes con RSC, destacando su alta especificidad y valor predictivo negativo. Así, este cuestionario puede ser aplicado en la consulta otorrinolaringológica diaria para evaluar la evolución del paciente durante el manejo de su patología rinosinusal

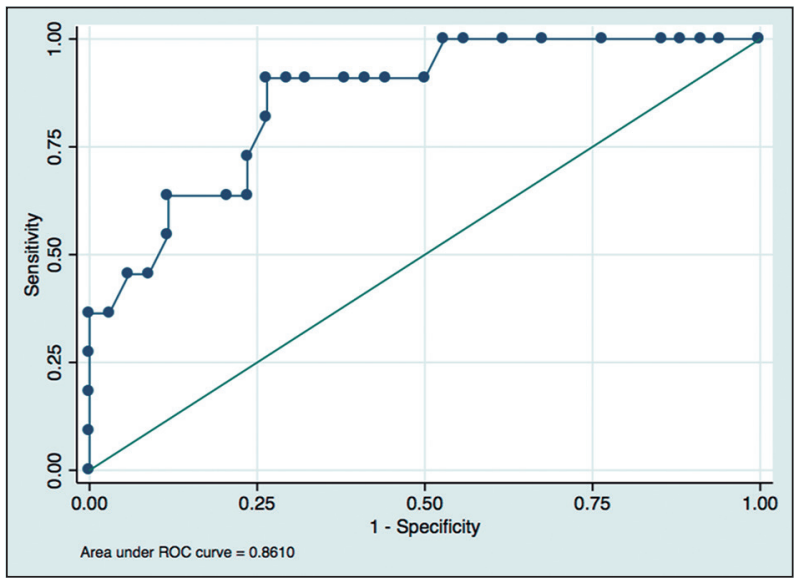

Figura 4. Validez discriminante. Área bajo la curva ROC de 0,861.

crónica. Por otro lado, al ser un cuestionario de autoaplicación, se facilita su respuesta previa a la consulta, sin interrumpir el proceso clínico del profesional. Por otro lado, es una herramienta útil para objetivar la sintomatología y realizar seguimiento de pacientes reclutados en trabajos de investigación futuros.

\section{Conclusión}

Esta traducción y adaptación transcultural del instrumento SNOT-22 es válida, confiable y concordante. Por lo tanto, es un buen instrumento para evaluar la severidad de los síntomas de RSC, la repercusión en la CdV de pacientes adultos chilenos y una herramienta para el seguimiento y control del tratamiento de estos pacientes. Adicionalmente, permite detectar y discriminar pacientes con RSC, con alta especificidad y valor predictivo negativo.

\section{Bibliografía}

1. Fokkens WJ, Lund VJ, Mullol J, et al. European Position Paper on Rhinosinusitis and Nasal Polyps 2012. Rhinol Suppl. 2012.

2. Bloom B, Cohen RA, Freeman G. Summary Health Statistics for U.S. children: National Health Interview Survey, 2011. Vital Heal Stat Ser 10 Data from Natl Heal Surv. 2012.

3. Hastan D, Fokkens WJ, Bachert C, et al. Chronic rhinosinusitis in Europe - An underestimated disease. 
A GA 2LEN study. Allergy Eur J Allergy Clin Immunol. 2011. doi:10.1111/j.1398-9995.2011.02646.x.

4. Collins JG. Prevalence of selected chronic conditions: United States, 1990-1992. Vital Health Stat 10. 1997.

5. Chen Y, Dales R, Lin M. The epidemiology of chronic rhinosinusitis in Canadians. Laryngoscope. 2003. doi:10.1097/00005537-200307000-00016.

6. Gliklich RE, Metson R. Effect of sinus surgery on quality of life. Otolaryngol - Head Neck Surg. 1997. doi:10.1016/S0194-5998(97)70199-2.

7. Gliklich RE, Metson R. The Health Impact of Chronic Sinusitis in Patients Seeking Otolaryngologic Care. Otolaryngol Head Neck Surg. 1995. doi:10.1016/ S0194-5998(95)70152-4.

8. Piccirillo JF, Merritt MG, Richards ML. Psychometric and clinimetric validity of the 20-Item Sino-Nasal Outcome Test (SNOT-20). Otolaryngol - Head Neck Surg. 2002. doi:10.1067/mhn.2002.121022.

9. Hopkins C, Slack R, Lund V, Brown P, Copley L, Browne J. Long-term outcomes from the english national comparative audit of surgery for nasal polyposis and chronic rhinosinusitis. Laryngoscope. 2009. doi:10.1002/lary.20653.

10. Asiri M, Alokby G. Validation and Cross-cultural Adaptation of the Sinonasal Outcome Test (SNOT)22 for the Arabian Patient Population. Cureus. 2019;11(4):e4447. doi:10.7759/cureus.4447.

11. Mozzanica F, Preti A, Gera R, et al. Cross-cultural adaptation and validation of the SNOT-22 into Italian. Eur Arch Otorhinolaryngol. 2017;274(2):887895. doi:10.1007/s00405-016-4313-x.

12. Numthavaj $\mathrm{P}$, Bhongmakapat $\mathrm{T}$, Roongpuwabaht $\mathrm{B}$, Ingsathit A, Thakkinstian A. The validity and reliability of Thai Sinonasal Outcome Test-22. Eur Arch Otorhinolaryngol. 2017;274(1):289-295. doi:10.1007/s00405-016-4234-8.

13. Adnane C, Adouly T, Oubahmane T, et al. Psychometric Validation of a Moroccan Version of the 22-Item Sino-Nasal Outcome Test. Otolaryngol Head Neck Surg. 2016;155(4):681-687. doi:10.1177/0194599816650477.

14. Shapira Galitz Y, Halperin D, Bavnik Y, Warman M. Sino-Nasal Outcome Test-22: Translation, Crosscultural Adaptation, and Validation in HebrewSpeaking Patients. Otolaryngol Head Neck Surg. 2016;154(5):951-956. doi:10.1177/0194599816629378.

15. de los Santos G, Reyes P, del Castillo R, Fragola C, Royuela A. Cross-cultural adaptation and validation of the sino-nasal outcome test (SNOT-22) for Spanish-speaking patients. Eur Arch Otorhinolaryngol. 2015;272(11):3335-3340. doi:10.1007/s00405-0143437-0.

16. de Dorlodot C, Horoi M, Lefebvre P, et al. French adaptation and validation of the sino-nasal outcome test-22: a prospective cohort study on quality of life among 422 subjects. Clin Otolaryngol. 2015;40(1):2935. doi:10.1111/coa.12315.
17. Lachanas VA, Tsea M, Tsiouvaka S, Hajiioannou JK, Skoulakis CE, Bizakis JG. The sino-nasal outcome test (SNOT)-22: validation for Greek patients. Eur Arch Otorhinolaryngol. 2014;271(10):2723-2728. doi:10.1007/s00405-014-2969-7.

18. Jalessi M, Farhadi M, Kamrava SK, et al. The reliability and validity of the persian version of sinonasal outcome test 22 (SNOT 22) questionnaires. Iran Red Crescent Med J. 2013;15(5):404-408. doi:10.5812/ircmj.7937.

19. Vaitkus S, Padervinskis E, Balsevicius T, et al. Translation, cross-cultural adaptation, and validation of the sino-nasal outcome test (SNOT)-22 for Lithuanian patients. Eur Arch Otorhinolaryngol. 2013;270(6):1843-1848. doi:10.1007/s00405-0122282-2.

20. Kosugi EM, Chen VG, Fonseca VMG da, Cursino MMP, Mendes Neto JA, Gregorio LC. Translation, cross-cultural adaptation and validation of SinoNasal Outcome Test (SNOT): 22 to Brazilian Portuguese. Braz J Otorhinolaryngol. 2011;77(5):663-669. doi:10.1590/s1808-86942011000500021.

21. Lu W, Qi F, Gao Z-Q, Feng G-D, Yuan X-D, Jin X-F. [Quality of life survey on patients with chronic rhinosinusitis by using Chinese version of the 22-item sinonasal outcome test (SNOT-22)]. Zhonghua Er Bi Yan Hou Tou Jing Wai Ke Za Zhi. 2008;43(1):18-21.

22. Schalek P, Otruba L, Hahn A. Quality of life in patients with chronic rhinosinusitis: A validation of the Czech version of SNOT-22 questionnaire. Eur Arch Oto-Rhino-Laryngology. 2010;267(3):473-475. doi:10.1007/s00405-009-1180-8.

23. Lange B, Thilsing T, Al-Kalemji A, Bælum J, Martinussen T, Kjeldsen A. The Sino-Nasal outcome Test 22 validated for Danish patients. Dan Med Bull. 2011;58(2).

24. Kosugi EM, Chen VG, da Fonseca VMG, Cursino MMP, Neto JAM, Gregório LC. Translation, crosscultural adaptation and validation of sinonasal outcome test (SNOT)-22 to Brazilian Portuguese. Braz J Otorhinolaryngol. 2011;77(5):663-669. doi:10.1590/S1808-86942011000500021.

25. Hopkins C, Gillett S, Slack R, Lund VJ, Browne JP. Psychometric validity of the 22-item Sinonasal Outcome Test. Clin Otolaryngol. 2009. doi:10.1111/ j.1749-4486.2009.01995.x.

26. Guillemin F, Bombardier C, Beaton D. Cross-cultural adaptation of health-related quality of life measures: Literature review and proposed guidelines. J Clin Epidemiol. 1993;46(12):1417-1432. doi:10.1016/08954356(93)90142-N.

27. Lawshe, C. (1975). Pruebas psicotécnicas en la selección de personal. Madrid: Rialp.

28. Kelinger F, Lee H. Foundations of behavioral research. USA: Harcourt Brace. 2002.

29. Aiken, Lewis R. Tests psicológicos y evaluación. 
Undécima edición, Pearson Educación, México, 2003 ISBN: 970-26-0431-1.

30. Argimon JM, Jiménez J. Validación de cuestionarios. Argimon J, Jiménez J. Métodos de investigación clínica y epidemiológica. Madrid: Elsevier 2004.

31. Carvajal A, Centeno C, Watson R, Martínez M, Sanz Rubiales Á. ¿Cómo validar un instrumento de medida de la salud? Anales del Sistema Sanitario de Navarra. 2011;34(1):63-72.

32. Rabanales A. El español de Chile: presente y futuro. Onomázein Rev Lingüística, Filol y Traducción. 2000;5:135-141.

33. Craddock JR, Oroz R. La lengua castellana en Chile. Hispania. 1968;51:600. 